\title{
Proceso de atención de enfermería a lactante con enfermedad huérfana, bajo el modelo de Callista Roy
}

\author{
Nursing Care Process of an Infant with an Orphan Disease under the \\ Callista Roy's Model
}

\section{O processo do cuidado em Enfermagem, à crianças lactante com doença órfã, sob o modelo de Callista Roy}

\author{
Shirley Brigthy Portilla-Ordoñez. * \\ Andrés Felipe Villarreal-Peñaloza. ** \\ Pilar Abreu-Peralta, Enf., Esp., MSc. ***
}

\begin{abstract}
Resumen
Introducción: Las enfermedades con diagnóstico no especificado son denominadas enfermedades huérfanas; definidas como aquellas por las que el enfermo puede encontrarse en peligro de muerte o de invalidez crónica y tienen una prevalencia de 1 caso por cada 5,000 personas. El Proceso de Atención de Enfermería (PAE) permite proporcionar una atención integral al sujeto de cuidado con estas patologías y su familia. Objetivo: Brindar cuidado holístico de enfermería a lactante con enfermedad huérfana, bajo el modelo de adaptación de Sor Callista Roy. Metodología: La selección del sujeto de cuidado se realiza en terapia de neurodesarrollo en febrero de 2015; con previo consentimiento informado de la madre se inicia análisis de caso en modalidad de seguimiento domiciliario, haciendo uso del método proceso de atención de enfermería bajo el enfoque del modelo de adaptación de Sor Callista Roy. Resultados:Al finalizar el proceso de atención de enfermería propuesto se pudo observar en la lactante y su familia: el mejoramiento de las respuestas ineficaces
\end{abstract}

detectadas con el modelo de Callista Roy, evidenciado por el aumento de peso, el fortalecimiento de los vínculos familiares, la generación de un ambiente social y recreativo positivo para la lactante y la motivación en la búsqueda de ayuda profesional en situaciones de conflicto de decisiones.Conclusiones: Mediante las intervenciones realizadas, se pudo evidenciar el progreso de la lactante durante el periodo de seguimiento. La articulación del método proceso de atención de enfermería, teorías de enfermería y las herramientas North American Nursing Diagnosis Association (NANDA), Nursing Outcomes Classification (NOC), Nursing Interventions Classification (NIC) NNN, permite un manejo holístico a los niños con enfermedades huérfanas. [Portilla-Ordoñez SB, VillarrealPeñaloza AF, Abreu-Peralta P. Proceso de atención de enfermería a lactante con enfermedad huérfana, bajo el modelo de Callista Roy. MedUNAB 2016; 19(2): 151-160]

Palabras Clave: Enfermería pediátrica; Procesos de enfermería; Diagnóstico de enfermería; Enfermería holística; Lactante.

* Estudiante de Enfermería, Práctica Electiva de Profundización Investigativa, Instituto del Corazón de Bucaramanga.

** Estudiante de Enfermería VII semestre, Universidad Autónoma de Bucaramanga, Santander, Colombia.

*** Enfermera, Especialista en Administración de Servicios de Salud, Magíster en Educación, Docente Programa de Enfermería, Universidad Autónoma de Bucaramanga, Santander, Colombia.

Autor de correspondencia: Shirley Brigthy Portilla Ordóñez, Práctica Electiva de Profundización Investigativa, Instituto del Corazón de Bucaramanga, Colombia. E-mail: sportillo@unab.edu.co.

Artículo recibido: 1 de agosto de $2016 \quad$ Aceptado: 26 noviembre de 2016 


\begin{abstract}
Introduction: Diseases with unspecified diagnosis are called orphan diseases; these are defined as those for which the patient may be in mortal danger or chronic disability, and have a prevalence of 1 case every 5,000 people. The Nursing Care Process allows providing a comprehensive care to the patient with these pathologies and his/her family. Objective: To provide a holistic nursing care to an infant with an orphan disease, under the Callista Roy's adaptation model. Methodology: The patient selection is carried out during the neurodevelopmental therapy in February of 2015; with the baby's mother authorization, the case analysis in mode of home follow-up starts by using the Nursing Care Process method (NCP) under the approach the Callista Roy's adaptation model. Results: At the end of the proposed Nursing Care Process, it could be observed and detected the improvement of ineffective responses by implementing Callista Roy's model with the infant and her mother, due to the fact that the baby gained weight; also she could strengthen her family ties because of the generation of a positive social and recreational environment, her mother was motivated to seek professional help in decision-making situations. Conclusions: By the interventions made, the progress of the infant during the follow-up period could be verified. The articulation of the Nursing Care Process method, Nursing theories and North American Nursing Diagnosis Association (NANDA), Nursing Outcomes Classification (NOC), Nursing Interventions Classification (NIC) NNN tools allow a holistic management of children with orphan diseases. [PortillaOrdoñez SB, Villarreal-Peñaloza AF, Abreu-Peralta $P$. Nursing Care Process of an infant with an orphan disease under the Callista Roy's model. MedUNAB 2016; 19(2): 151160]
\end{abstract}

Keywords: Pediatric nursing; Nursing process; Nursing diagnosis; Holistic nursing; Infant.

\section{Introducción}

Se consideran enfermedades huérfanas o con diagnóstico no especificado, a aquellas que ponen al individuo en peligro de muerte o discapacidad crónica, con una prevalencia menor de 5 casos por cada 10,000 habitantes en la comunidad (1). El término "Enfermedades Huérfanas" comprende tanto las enfermedades raras como las olvidadas, consideradas "huérfanas" de atención por parte de la investigación, la industria farmacéutica y el estado con las políticas de salud pública (2).

El $80 \%$ de estas enfermedades son de origen genético y el otro $20 \%$ pueden ser congénitas (adquiridas en la vida intrauterina), autoinmunes (las células inmunológicas atacan el propio organismo), de origen infeccioso o simplemente aparecer de manera espontánea por diversos factores, incluyendo ambientales (3). Se cree que la mayoría de estas enfermedades se originan por mutaciones en uno o más de sus genes, muchas de estas mutaciones genéticas pueden heredarse, lo que explicaría el motivo del porqué muchas de estas enfermedades son familiares. Es importante saber que la genética es solo un factor.

\section{Resumo}

Introdução: As doenças com o diagnóstico não especificado são chamadas doenças órfãs; definidas como aquelas em que o paciente pode estar em risco de vida ou invalidez crônica e tem uma prevalência de 1 caso por cada 5.000 pessoas. O Processo do cuidado na Enfermagem pode proporcionar uma atenção integral ao sujeito com estas patologias e a sua família. Objetivo: prestar o cuidado holístico de enfermagem para crianças com doença órfã sob o modelo de adaptação da irmã Callista Roy. Metodologia: A seleção do sujeito do cuidado é feita na terapia do desenvolvimento neurológico em fevereiro de 2015, com o consentimento informado da mãe; a análise do caso começou com o monitoramento em casa, usando o método do processo dos cuidados de enfermagem (PAE), sob a adaptação do modelo de abordagem da Irmã Callista Roy. Resultados: No final do processo proposto dos cuidados de enfermagem, foi possível observar na criança e sua família; que melhorou as respostas ineficazes detectadas com o modelo de Callista Roy, foi visível no aumento de peso, no fortalecimento dos laços familiares, na geração do ambiente social e recreativo positivo para a criança e a motivação para buscar ajuda profissional em situações de conflito e toma de decisões. Conclusões: Através de intervenções feitas, foi possível evidenciar o progresso da criança durante o período de monitoramento. Ao combinar o método, Processo do Cuidado de Enfermagem, as teorias da Enfermagem e as ferramentas NNN, permitiram ocuidado holístico das crianças com doenças órfãs. [Portilla-Ordoñez SB, VillarrealPeñaloza AF, Abreu Peralta P. O processo do cuidado em Enfermagem, à crianças lactante com doença órfã, sob o modelo de Callista Roy. MedUNAB 2016; 19(2): 151-160]

Palavras-chave: Enfermagem pediátrica; Processos de enfermagem; Diagnóstico de enfermagem; Enfermagem holística; Lactente.

Existen otros factores externos como el tipo de alimentación, tabaco, exposición a químicos o radiaciones; los cuales influyen y pueden originar cambios durante la expresión genética y desarrollo de órganos. Estos factores pueden causar enfermedades o interactuar con factores genéticos para aumentar la severidad de una enfermedad. Cada ser humano es portador aproximadamente de 8 a 10 anormalidades genéticas las cuales generalmente son recesivas, lo que evita la manifestación de alteraciones o anormalidades físicas y/o conductuales (1). Por el contrario, en caso de que dos personas posean en su carga genética la misma anormalidad y estos individuos se unan para engendrar, se pueden ver afectados por alguna complicación dado el efecto agonista de la unión de los genes.

En el mundo se han identificado entre 6,000 y 7,000 enfermedades huérfanas, las cuales aumentan de manera progresiva a pesar de que su número ya es muy elevado. En Colombia se denomina enfermedades huérfanas a aquellas con una prevalencia menor de 1 caso por cada 5,000 personas (4), se encuentran identificadas alrededor de 1,920 enfermedades de este tipo y 13,168 pacientes diagnosticados con alguna de estas enfermedades, las cuales se encuentran incluidas en la resolución 430 de 2013 de Colombia (5). 
Las enfermedades huérfanas son generalmente multisistémicas, crónicas, a menudo degenerativas y con alto riesgo de discapacidad y/o muerte (2). Tanto el paciente como su familia se ven enfrentados a dificultades que surgen directamente por la rareza de la patología; como consecuencia, quienes padecen este tipo de enfermedades poco frecuentes tienen dolencias diferentes, pero carencias comunes en cuanto a los pocos servicios de salud, falta de acceso al diagnóstico correcto, poca información, poca evidencia científica, escaso apoyo social, deteriorada calidad de vida, altos costos en servicios médicos y farmacéuticos, desigualdad en disponibilidad de tratamientos y cuidado profesional (6).

Las opciones diagnósticas y terapéuticas de estas enfermedades han evidenciado una mejoría significativa en el pronóstico, pero los pacientes tardan entre 5 y 10 años en obtener un diagnóstico del problema que sufren por la escasa información de mecanismos fisiopatológicos y bases moleculares de las enfermedades (7). Además, la no suficiente preparación en la formación, actuación y la poca experiencia que tienen los profesionales de la salud, en el cuidado al niño y a su familia, hace que se comprometa aún más la familia, comunidad y sociedad. En la lista de necesidades prioritarias para estos pacientes se destacan, la creación de centros especializados para diagnosticarlas y la generación de investigación con el objetivo de encontrar un tratamiento(1).

Dadas todas las dificultades que pueden atravesar estos pacientes y su familia se genera la necesidad de que enfermería actúe como promotora de cuidado, haciendo uso de herramientas como las guías de buenas prácticas clínicas, con evidencias que fortalezcan el campo de acción de la disciplina (8). El profesional de enfermería además de contar con guías basadas en la evidencia las cuales permiten proporcionar cuidado eficaz y con el menor riesgo posible a sus sujetos de cuidado, cuenta con modelos teóricos que permiten describir, explicar, predecir y controlar los fenómenos de la práctica de enfermería, guiando el proceso de atención de enfermería, con el cual se aplica en la práctica la teoría o modelo, que orienta y da sentido a dicha práctica.

Callista Roy, con experiencia pediátrica, reconoce la flexibilidad que poseen los niños y la capacidad de adaptación a los cambios físicos y fisiológicos importantes (9). En su modelo postula que los individuos tienen cuatro modos o métodos de adaptación: fisiológico, autoconcepto, desempeño del rol y relaciones de interdependencia, que son modificados por estímulos focales, contextuales y residuales. Los cuales orientan el cuidado para buscar condiciones que faciliten el logro de las metas personales del individuo: supervivencia, crecimiento, reproducción y dominio del individuo (10). El objetivo del seguimiento es brindar cuidado holístico de enfermería a lactante con enfermedad huérfana, bajo el modelo de adaptación de Sor Callista Roy.

\section{Metodología}

Para el reporte de caso se desarrolla el método Proceso de Atención de Enfermería (PAE), mediante la selección de un paciente en práctica formativa del curso cuidado holístico al niño y adolescente. La selección del sujeto de cuidado se realiza en terapia de neurodesarrollo en febrero de 2015; con previo consentimiento informado de la madre se inicia análisis de caso en modalidad de seguimiento domiciliario durante un periodo de 4 meses. Lavaloración de la lactante se efectúa según el modelo de adaptación de Sor Callista Roy, con el cual se identifican las respuestas ineficaces prioritarias de la lactante y acto seguido se plantean los diagnósticos de enfermería, sus objetivos e intervenciones con sus respectivas actividades usando las herramientas taxonómicas North American Nursing Diagnosis Association (NANDA) (11), Nursing Outcomes Classification (NOC) (12), Nursing Interventions Classification(NIC) (13).

Considerando los aspectos éticos establecidos por el Tribunal Nacional Ético de Enfermería de Colombia en la ley 911 de 2004, se establece la responsabilidad deontológica para el ejercicio de la profesión de enfermería en Colombia (14). Este seguimiento es clasificado como "investigación de riesgo mínimo" de acuerdo a la resolución 8430 del 4 de octubre de 1993 del Ministerio de Protección Social de Colombia (15), se mantendrá confidencialidad de los datos personales de los sujetos de cuidado, y se solicitará el consentimiento informado por parte del sujeto de cuidado o responsable legal en este caso su cuidador principal.

\section{Presentación del caso}

Lactante mayor de sexo femenino con 20 meses de edad y 3 meses de edad mental, quien vive con sus padres en un barrio de Bucaramanga (Colombia), se encuentra en terapia de neurodesarrollo en compañía de su madre, antecedentes perinatales: prematuridad (30.5 semanas de edad gestacional), síndrome de dificultad respiratoria, con peso al nacer: 910 gr y talla: $40 \mathrm{~cm}$, grupo sanguíneo $\mathrm{O}+$. Recibe terapia física seis días a la semana. Es valorada por diferentes especialidades como: pediatría, genética, neurología, ortopedia, neumología, neurodesarrollo, nutrición. Diagnóstico médico: sin especificar (enfermedad huérfana) presenta hipotonía muscular generalizada, miopatía congénita, retardo del crecimiento y desarrollo.

Neurodesarrollo: Moderada activación muscular asociada a su hipotonía central, con moderada coactivación de músculos agonistas y antagonistas afectando la velocidad en la ejecución del movimiento consecuente con su debilidad muscular central. Débil control cefálico. Hipotonía orofacial e inconsistencia en las praxias linguales y faciales. Miembros superiores semiflejados, con agarre a mano llena y pinza dependiendo del tamaño del objeto, eleva miembros inferiores despegándolos de la base de soporte, realiza 
rolados y mantiene posición de arrodillado por 1 minuto con asistencia.

\section{Valoración según el modelo de adaptación de Sor Callista Roy}

En enfermería, los modelos conceptuales constituyen un marco de referencia para la práctica, proporcionando una guía acerca de cómo observar e interpretar las respuestas del paciente en una situación de enfermedad. Roy resalta que los seres humanos son sistemas adaptativos holísticos, que funcionan con partes interdependientes y actúan en unidad de propósito, no en respuesta causa-efecto. Los seres humanos, como seres adaptativos, tienen la capacidad de adaptarse y crear cambios en el ambiente. La habilidad para responder a estos cambios está determinada por las demandas de la situación y los recursos internos disponibles (10).

Para Callista Roy los sujetos de cuidado cuentan con 4 modos de adaptación (fisiológico, autoconcepto, interrelación y función del rol) los cuales se ven condicionados por 3 clases de estímulos (focales, contextuales, residuales). Enfermería participa en la modificación de estos estímulos buscando que el sujeto se adapte al cambio, y que su familia se convierta en un receptor de cuidados, por medio de un proceso de aprendizaje que le permita modificar las respuestas ineficaces.

\section{Estímulos}

Focales: distonia muscular generalizada, hipotonía central, retraso en el crecimiento y desarrollo, aislamiento social.

Contextuales: alimentación por sonda de gastrostomía, conflictos familiares, escasa interacción con el entorno.

Residuales: cuidadora que no busca información profesional y de acuerdo a sus creencias toma decisiones.

\section{Modos de adaptación}

\section{Función fisiológica:}

1.1) Nutrición: peso: $8,2 \mathrm{~kg}$, Talla: $87 \mathrm{~cm}$. Inadecuada relación peso/talla dada por un índice de masa corporal de 10,83 (Figura 1 y Figura 2). Dieta hiperproteica más alimentación complementaria, de consistencia liquida administrada por sonda de gastrostomía.

1.2) Eliminación: sistema urinario: poliuria (8-10 micciones diarias), orina espontánea en pañal, de color y olor normal. Sistema gastrointestinal: eliminación fecal 1 vez al día, día por medio, de aspecto normal. La madre refiere ayudar a la lactante a realizar deposiciones cambiándola de posiciones. 1.3) Líquidos y electrolitos: ingiere 1 litro de agua al día por medio de la gastrostomía, la piel se encuentra normo hidratada, con adecuada turgencia.

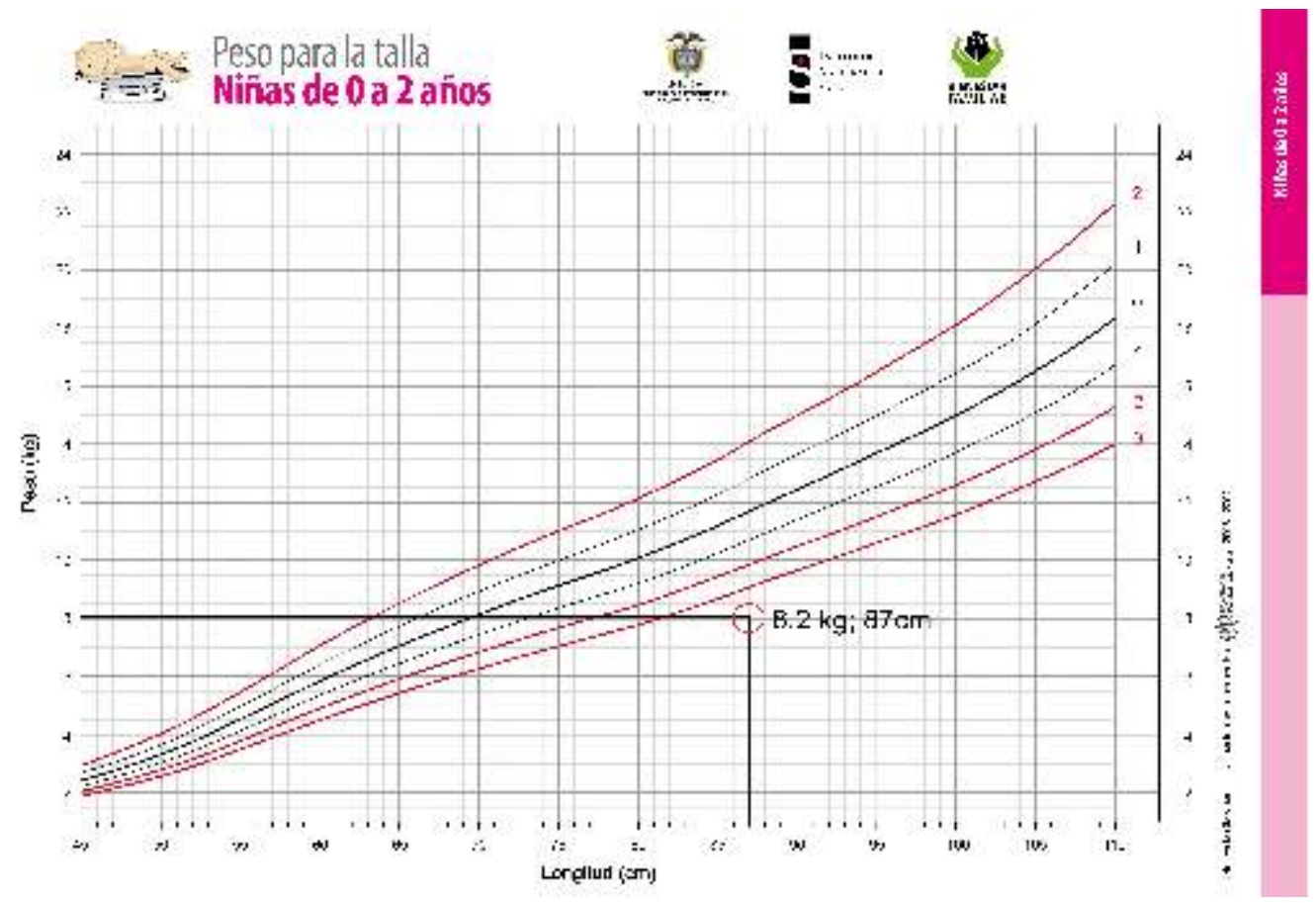

Fuente: tablas de crecimiento y desarrollo AIEPI, relación peso para la talla en menores femeninos < 2 años. Organización Mundial de la Salud (OMS) (16).

Figura 1. Relación peso/talla de la lactante a sus 20 meses de edad por debajo de la desviación estándar -3, muy bajo peso para la talla. 


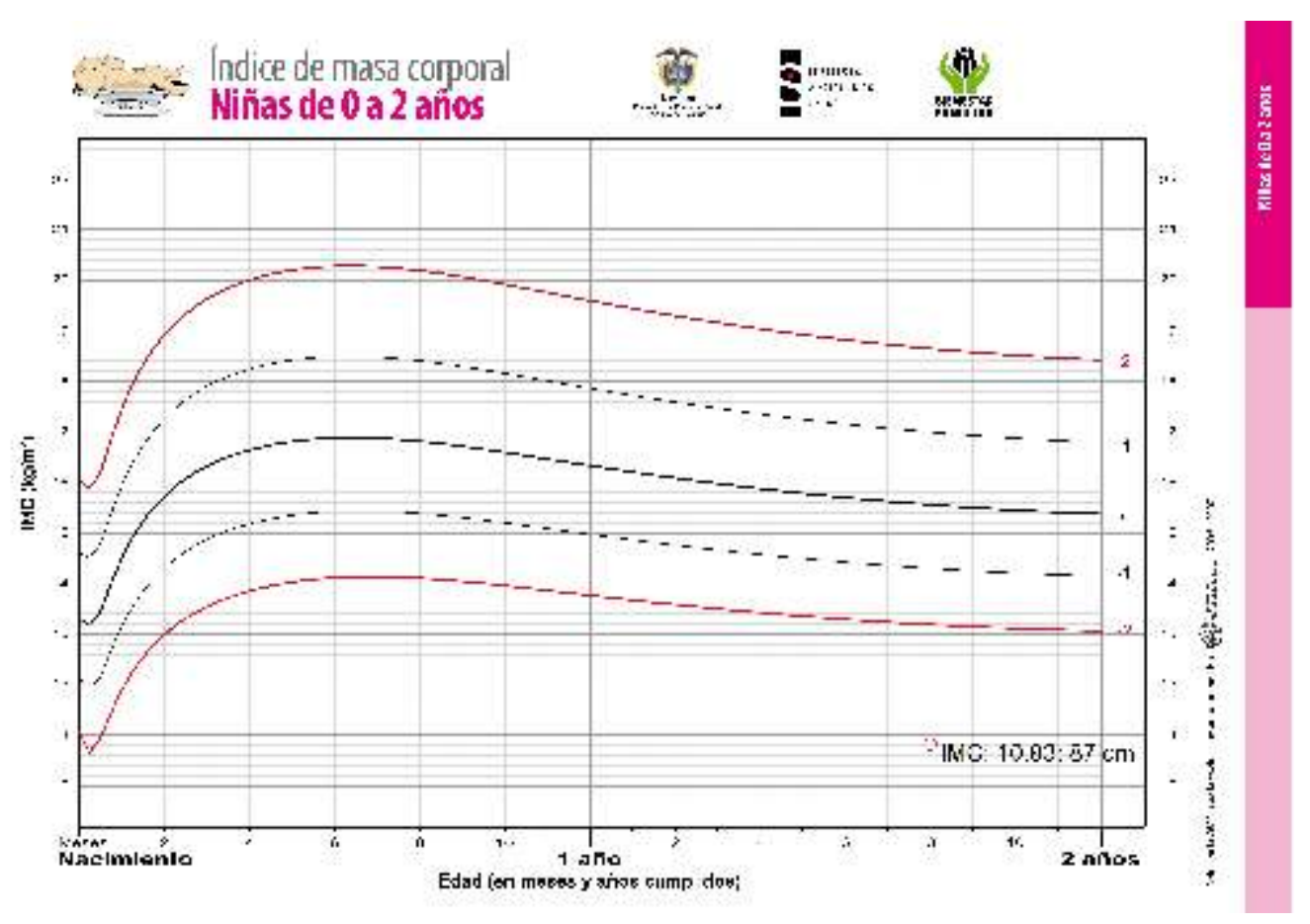

Fuente: tablas de crecimiento y desarrollo AIEPI, índice de masa corporal en menores femeninos <2 años. Organización Mundial de la Salud (OMS) (16).

Figura 2. Índice de masa corporal de la lactante a sus 20 meses de edad por debajo de la desviación estándar-2, desnutrición severa.

1.4) Oxígeno y circulación: frecuencia cardiaca: 95 latidos por minuto, rítmicos, frecuencia respiratoria: 38 respiraciones por minuto, respiración espontánea, toracoabdominal, a la auscultación sin presencia de ruidos sobreagregados, no hay evidencia de trabajo respiratorio forzado, llenado capilar $<3$ segundos, sin evidencia de edemas en extremidades.

1.5) Integridad cutánea: la piel se encuentra limpia, íntegra, hidratada, normo térmica temperatura: $35.8^{\circ} \mathrm{C}$; los pies íntegros sin lesiones; en flanco izquierdo inserción de gastrostomía, zona integra sin eritema o signos de infección. 1.6) Ejercicio y descanso: duerme entre 8-10 horas diarias, hipotonía muscular generalizada, leve control cefálico, no hay control sedente. Evaluación fuerza muscular según la escala de fuerza muscular modificada (MRC) la lactante se encuentra en grado 3- (movimiento activo que sólo puede realizarse de forma parcial contra la gravedad), deterioro de la deambulación, se le dificulta mantener una posición de pie, generalmente se mantiene posición prono o sedente con ayuda.

\section{Autoconcepto:}

2.1) Identidad física: desarrollo neurológico de la niña no es idóneo para su edad cronológica, su edad mental es de 3 meses y su edad cronológica de 20 meses, se evidencia retraso en el crecimiento y desarrollo, además de sus funciones motoras y cognitivas; se evidencia dificultad de la lactante para realizar actividades correspondientes a su edad.
2.2) Identidad personal: no realiza actividades de una niña de 20 meses como: gatear/caminar, ingerir y deglutir alimentos por vía oral, hablar, jugar o relacionarse con niños de su grupo etario.

2.3) Identidad moral y ética: inmadura no cuenta con una capacidad de juicio plena para tomar decisiones morales, condicionado por la escasa relación con el entorno y la etapa evolutiva en que se encuentra, en la que requiere una mayor interacción con niños del mismo grupo etario y sus familiares. Con un nivel bajo de responsabilidad moral debido a su situación de discapacidad, se evidencian respuestas físicas que respaldan la capacidad de reconocer a su alrededor situaciones u objetos que le interesan y llaman la atención, o por lo contrario le causan disgusto y desagrado.

\section{Función de rol:}

3.1) Rol primario: niña, 20 meses de edad, 3 meses edad mental.

3.2) Rol secundario: lactante mayor, hija, nieta, prima.

3.3) Rol terciario: paciente con enfermedad huérfana, hipotonía muscular generalizada, aislamiento social.

\section{Interdependencia:}

4.1) Conductas dependientes: requiere ayuda de su madre para realizar todas las actividades que demandan movimientos físicos debido a su hipotonía con hipotrofia muscular generalizada, recibe cuidados 24 horas del día por parte de sus padres, no se cuenta con apoyo familiar en los cuidados diarios de la lactante, la cuidadora manifiesta requerir apoyo. Se denotan problemas a nivel cognitivo y 
retraso en el crecimiento y desarrollo de la paciente debido a la complejidad de su patología, por esta situación la paciente es totalmente dependiente de la madre.

4.2) Conductas independientes: debido a la patología que presenta la menor, no puede tomar iniciativa en actividades motoras o interactuar con su entorno.

\section{Relaciones aplicadas según el modelo teórico}

Teniendo en cuenta los estímulos focales, contextuales y residuales se establecen mecanismos de afrontamiento que llevan a enfermería a relacionarse con el sujeto de cuidado, para modificar su entorno y generar sistemas de adaptación, tanto en la paciente como en la familia, logrando la activación de un proceso de aprendizaje. Se establecen así, dos tipos de relación: familia-enfermero y enfermeropaciente, generando la modificación de las respuestas ineficaces y creando estrategias de control que favorecen la adaptación al cambio.

\section{Proceso de Atención de Enfermería}

Para llevar a cabo el proceso de atención de enfermería y tomando como referencia el modelo de adaptación planteado por Callista Roy, se implementó un plan de cuidados con las interrelaciones NANDA, NOC y NIC. Se identificaron 4 diagnósticos de enfermería los cuales fueron intervenidos durante el período de seguimiento.

Diagnóstico 1: desequilibrio nutricional: ingesta inferior a las necesidades (00002), relacionado con incapacidad para digerir los alimentos, manifestado por peso corporal inferior en un $41 \%$ de su peso ideal, con un percentil de -3 en tabla de peso para la talla (Figura 1). Bajo tono muscular, debilidad de los músculos requeridos para la masticación o la deglución.

Fundamentación: en las enfermedades neuromusculares, perder peso conlleva también el riesgo de perder masa muscular, mientras que ganar peso implica sobre todo un aumento de grasa. Para evitar la desnutrición proteica y las modificaciones de las necesidades energéticas ligadas a la enfermedad neuromuscular, es importante un seguimiento estricto profesional (17).

Evaluación: estado nutricional (1045) NOC inicial 50\% llevándolo a NOC final del $55 \%$ por medio de la intervención manejo de la nutrición (1100), (Tabla 1).

Diagnóstico 2: aislamiento social (00053), relacionado con factores contribuyentes a la ausencia de relaciones satisfactorias (retraso en el logro de las tareas del desarrollo),

Tabla 1. Plan de cuidados, indicadores de resultados e intervenciones.

Etiqueta diagnóstica: Desequilibrio nutricional: ingesta inferior a las necesidades (00002)

Dominio 2: Nutrición Clase 1: Ingestión

Resultado NOC: Estado nutricional (1045)

Escala likert: desviación grave del rango normal (1) hasta sin desviación del rango normal (5)

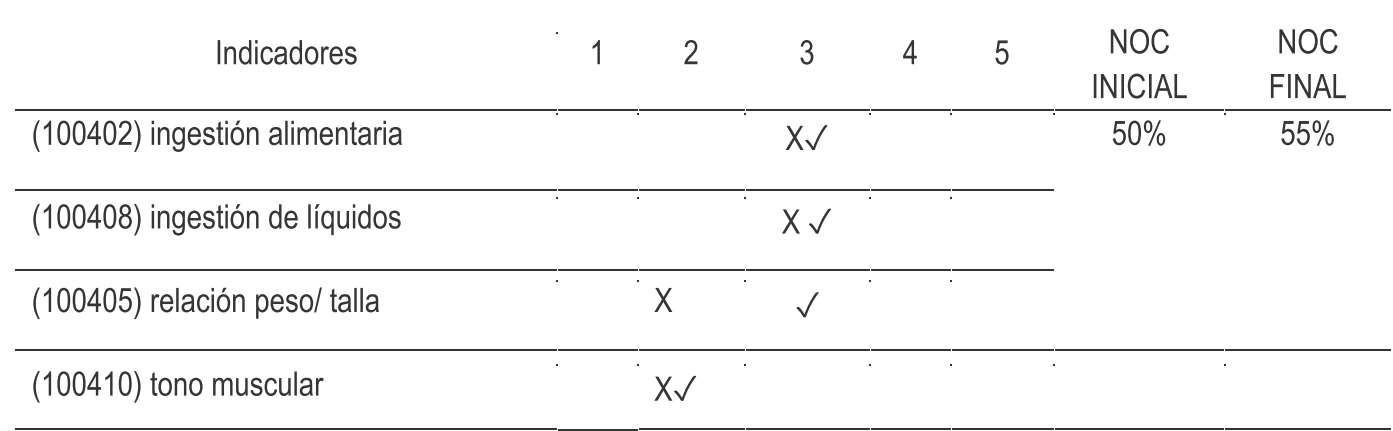

Intervención NIC: Manejo de la nutrición (1100)

ACTIVIDADES

- Proporcionar información adecuada acerca de necesidades nutricionales y modo de satisfacerlas

- Determinar en colaboración con el dietista, si procede el número de calorías y tipo de nutrientes necesarios para satisfacer las exigencias de alimentación

- Fomentar la ingesta de calorías adecuadas al tipo corporal y estilo de vida

- Vigilar tendencias de pérdida de peso y ganancia de peso

Fuente: elaboración propia de los autores 
Tabla 2. Plan de cuidados, indicadores de resultados e intervenciones.

\begin{tabular}{|c|c|c|c|c|c|c|c|}
\hline \multicolumn{8}{|c|}{$\begin{array}{l}\text { Etiqueta diagnóstica: Aislamiento social (00053) } \\
\text { Dominio 12: confort Clase 2: confort social }\end{array}$} \\
\hline \multicolumn{8}{|c|}{ Resultado NOC: Participación en juegos (0116) } \\
\hline \multicolumn{8}{|c|}{ Escala likert : desde nunca demostrado (1) hasta siempre demostrado (5) } \\
\hline Indicadores & 1 & 2 & 3 & 4 & 5 & $\begin{array}{l}\text { NOC } \\
\text { INICIAL }\end{array}$ & $\begin{array}{l}\text { NOC } \\
\text { FINAL }\end{array}$ \\
\hline (11601) Participación en juegos & & & $x$ & $\checkmark$ & & $50 \%$ & $70 \%$ \\
\hline (11603) Expresión de placer con el juego & & & $x$ & $\checkmark$ & & & \\
\hline $\begin{array}{l}\text { (11607) Expresión de emociones durante } \\
\text { el juego }\end{array}$ & & $x$ & $\checkmark$ & & & & \\
\hline (11608) Uso del rol del juego & & $x$ & $\checkmark$ & & & & \\
\hline \multicolumn{8}{|c|}{ Intervención NIC: Participación en juegos (0116) } \\
\hline $\begin{array}{l}\text { ACTIVIDADES } \\
\begin{aligned}- & \text { Animar al cuidador a que la lacta } \\
- & \text { Fomentar las relaciones con niño } \\
- & \text { Fomentar actividades sociales } \\
- & \text { Proporcionar juegos adecuados }\end{aligned}\end{array}$ & que & olle & ione & jjetiv & com & & \\
\hline
\end{tabular}

Fuente: elaboración propia de los autores

manifestado por conductas inadecuadas para la etapa de desarrollo, enfermedad, evidencia de discapacidad (física, mental).

Fundamentación: el menor para ampliar cada vez más su vida social necesitará la aprobación del grupo para la construcción y reafirmación de una autoimagen positiva. Estar enfermo es negativo y comprende ser perjudicial, no deseable y socialmente devaluado. Por lo general, las comparaciones causan baja autoestima y sentimientos de discriminación. La relación con los niños de la misma edad, ayudará a desarrollar su autoconcepto y la autoestima (18).

Evaluación: participación en juegos (0116) NOC inicial $50 \%$ llevándolo a NOC final del $70 \%$ por medio de la intervención potenciación de la socialización (5100), (Tabla 2)

Diagnóstico 3: afrontamiento familiar incapacitante (00073), relacionado con relaciones familiares muy ambivalentes, manifestado por asumir los signos de enfermedad del cliente y conductas familiares que son perjudiciales para el bienestar.

Fundamentación: la enfermedad del niño es siempre un problema familiar y su calidad de vida está íntimamente relacionada con el apoyo familiar que recibe. Los padres tienen que adaptarse a las características de cada enfermedad en sus diferentes fases (diagnóstico, tratamientos...) restructurando su vida familiar. La enfermedad puede ser impredecible, requiere atención constante y llegar a formar parte de la vida familiar, produciendo cambios estructurales, procesuales y emocionales en la familia (19). Evaluación: afrontamiento de los problemas de la familia (2600) NOC inicial $60 \%$ llevándolo a NOC final del $75 \%$ por medio de la intervención fomentar la implicación familiar (7110), (Tabla 3).

Diagnóstico 4: conflicto de decisiones (00083), relacionado con fuentes de información divergentes, manifestado por vacilación entre elecciones y alternativas verbaliza incertidumbre sobre las elecciones.

Fundamentación: las consultas telefónicas permiten aclarar dudas de los padres sobre las necesidades de consulta; existe disponibilidad inmediata de profesionales capacitados, como la enfermera; permite conocer la evolución del paciente; ayuda a los padres a manejar las quejas menores; evita visitas innecesarias al consultorio; promueve el uso de tratamientos caseros; permite clasificar las quejas de los pacientes, logrando la decisión más adecuada en cada caso; permite detectar síntomas de peligro para una atención precoz; y permite a los padres aclarar dudas de consultas anteriores (20). 
Tabla 3. Plan de cuidados, indicadores de resultados e intervenciones.

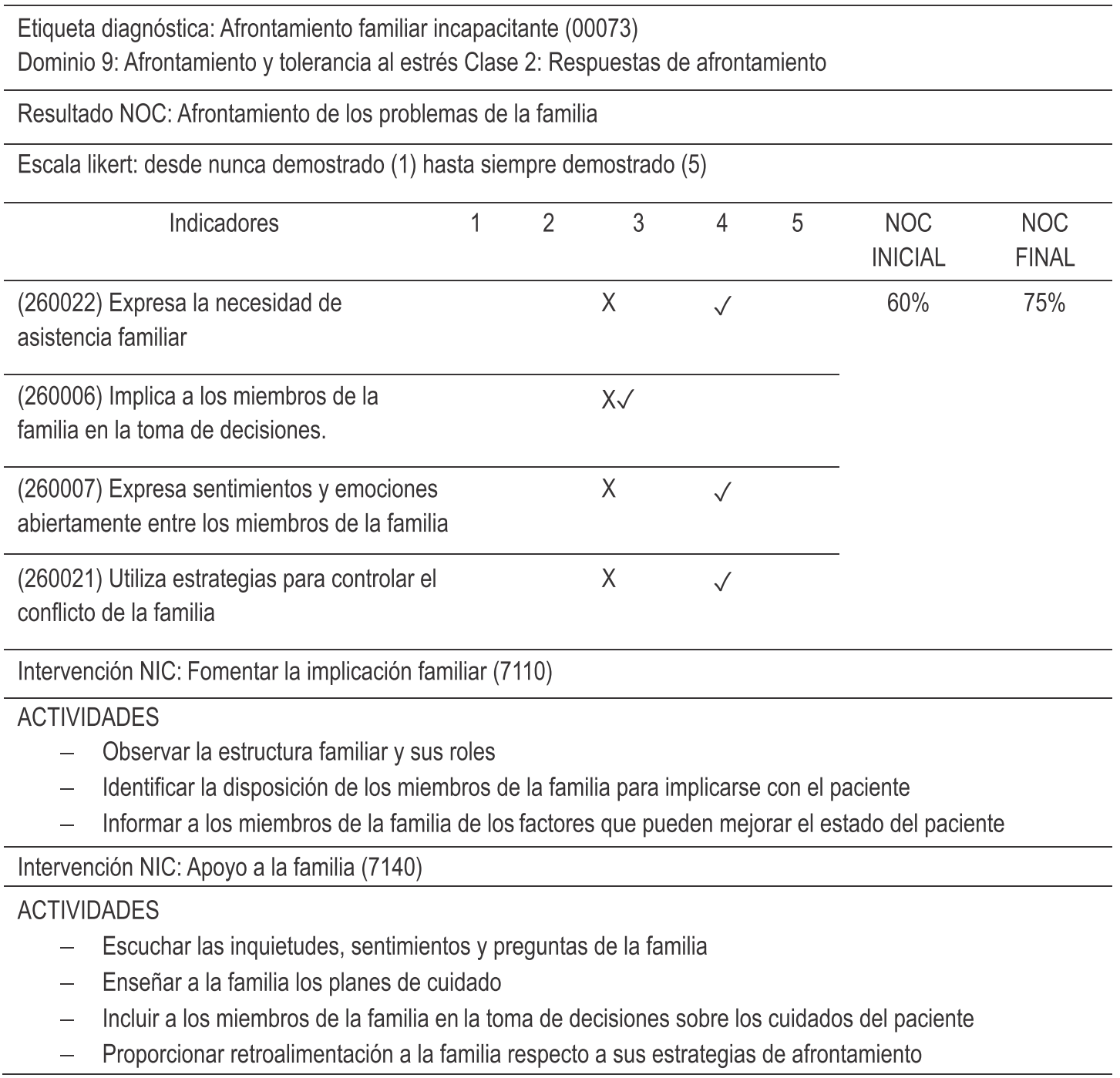

Fuente: Elaboración propia de los autores

Evaluación: toma de decisiones (0906) NOC inicial 72\% llevándolo a NOC final del $92 \%$ por medio de la intervención consulta por teléfono (8180), (Tabla 4).

\section{Resultados}

El seguimiento a la lactante comprendió 4 meses, en los cuales se realizaron 7 visitas domiciliarias con la asesora para evaluar los resultados del seguimiento, evidenciado por un NOC inicial general de 58\% llevado a un NOC final general de 73\% (sumando los 4 NOC inicial y final obtenidos, divididos por los 4 diagnósticos). Con base en las respuestas ineficaces detectadas con el modelo de Callista Roy se logró instaurar en la lactante una nueva dieta acorde a sus necesidades calóricas, con la cual se obtuvo un aumento de 400 gr de peso.
Se llevó a la familia a entender el proceso de enfermedad de la lactante y la importancia de generar un ambiente social positivo con niños de su mismo grupo etario, evitando el aislamiento social y la adaptación al entorno en la lactante. Recalcando la importancia tanto para la paciente como para sus cuidadores de generar un ambiente social amigable, debido a la afectación social que conlleva tener un familiar con una enfermedad de este tipo.

Se logró el fortalecimiento de los roles y relaciones familiares, por medio de terapia familiar individual y grupal, logrando mayor compromiso familiar en los cuidados de la lactante. Se generó el apoyo a la cuidadora principal, en este caso la madre en las horas de cuidados y acompañamiento, con el fin de prevenir un estrés por sobrecarga.

Se propició en la madre la búsqueda de apoyo en presencia de complicaciones o crisis de la lactante, se brindó asesoría, 
Tabla 4. Plan de cuidados, indicadores de resultados e intervenciones.

Etiqueta diagnóstica: Conflicto de decisiones (00083)
Dominio 10: Principios Vitales Clase 3: Congruencia entre valores/creencias/acciones

Resultado NOC: Toma de decisiones (0906)

Escala likert: desde gravemente comprometido (1) hasta no comprometido (5)

\begin{tabular}{|c|c|c|c|c|c|c|c|}
\hline Indicadores & 1 & 2 & 3 & 4 & 5 & $\begin{array}{c}\text { NOC } \\
\text { INICIAL }\end{array}$ & $\begin{array}{l}\text { NOC } \\
\text { FINAL }\end{array}$ \\
\hline (90601) Identifica información relevante & & & $\mathrm{x}$ & $\checkmark$ & & $72 \%$ & $92 \%$ \\
\hline (90602) Identifica alternativas & & & & $x$ & $\checkmark$ & & \\
\hline $\begin{array}{l}\text { (90604) Identifica recursos necesarios para } \\
\text { apoyar cada alternativa }\end{array}$ & & & $\mathrm{x}$ & $\checkmark$ & & & \\
\hline (90608) Compara alternativas & & & & $x$ & $\checkmark$ & & \\
\hline (90609)Escoge entre varias alternativas & & & & $x$ & $\checkmark$ & & \\
\hline \multicolumn{8}{|l|}{ Intervención NIC: Consulta por teléfono (8180) } \\
\hline 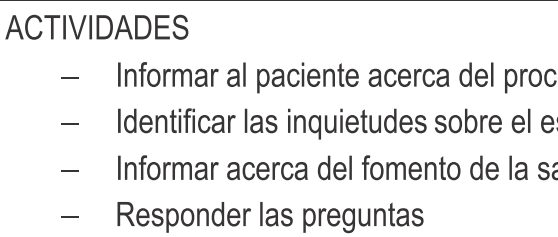 & $\begin{array}{l}\text { e las } \\
\text { de } \\
\text { educ }\end{array}$ & nad & $\begin{array}{l}\text { stene } \\
\text { salud }\end{array}$ & con & timic & & \\
\hline
\end{tabular}

Fuente: Elaboración propia de los autores

apoyo asistencial, y se educó en el uso de las Tecnologías de la Información y la Comunicación (TIC) para la búsqueda de ayuda familiar $\mathrm{y} / \mathrm{o}$ profesional, con el fin de disminuir el riesgo y prevenir eventos adversos.

\section{Conclusiones}

El proceso de atención de enfermería es un método sistemático compuesto por cinco etapas: valoración, diagnóstico de enfermería, planeación del cuidado, ejecución del mismo y evaluación de los resultados. En articulación con la taxonomía North American Nursing Diagnosis Association (NANDA), Nursing Outcomes Classification (NOC), Nursing Interventions Classification (NIC). Permite al enfermero(a) proporcionar atención integral a sus sujetos de cuidado y sus familias, con la posibilidad de llegar a sus hogares.

El modelo de Callista Roy permite describir, explicar, predecir y controlar los fenómenos de la práctica de enfermería, en la generación de los cuidados a la población pediátrica, la atención integral y seguimiento a sus sujetos de cuidado en estado de salud o enfermedad.
Sujetos de cuidado como los niños con enfermedades huérfanas, que representan un relevante problema de salud pública con alto grado de complejidad, curso clínico crónico y progresivamente debilitante. Requieren de un abordaje multidisciplinario, de actuación y seguimiento de enfermería; con el fin de disminuir su mortalidad, mejorar el acceso a los servicios de salud, proporcionar un tratamiento oportuno y eficaz para controlar los síntomas y mejorar la calidad de vida de estos pacientes.

\section{Conflicto de intereses}

Los autores manifiestan que no tienen ningún conflicto de interés.

\section{Referencias}

1. Lugones-Botell M, Ramírez-Bermúdez M. Enfermedades raras. Rev Cubana Med Gen Integr 2012; 28(3): 340-350

2. González-Vega L, Hernández-Córdova G, Solórzano S. Enfermedades huérfanas en pediatría: a propósito del día mundial de las enfermedades raras. RevMed Chile 2013; 141(2): 270-271. 
3. Cely-Galindo G, Gutiérrez SY. Reflexiones bioéticas en torno a las enfermedades raras y huérfanas con impacto en el sistema bucodentario. Revlatinoambioet 2011, 11(1): 22-31. Disponible en: http://www.scielo.org.co/pdf/ rlb/v11n1/v11n1a03.pdf

4. Ley $1438 / 2011$ de 19 de enero. Reforma del sistema general de seguridad social y se dictan otras disposiciones. (Instituto Nacional de Salud, artículo 140). Disponible en: http://www.ins.gov.co/normatividad/ Leyes/LEY\%201438\%20DE\%202011.pdf

5. Resolución $0430 / 2013$ de 20 de febrero. Listado de las Enfermedades Huérfanas. (Ministerio de salud y protección social). Disponible en: http://www.fecoer.org/ download/Resoluci\%C3\%B3n\%200430\%20de\%20201 3.pdf

6. European Organisation for Rare Diseases. Enfermedades Raras: El conocimiento de esta prioridad de la salud pública. 2005. [Acceso 02 de agosto 2016]. Disponible en: http://www.eurordis.org//MG/ pdf/Princeps_document-SN.pdf

7. Ministerio de salud y protección social. Protocolo de vigilancia en salud pública. Enfermedades Huérfanas-Raras. 2016; versión 02:1-23. Disponible en: http://www.fecoer.org/download/PRO\%20Enfermedade s\%20huerfanas $\% 20 y \% 20$ raras(3).pdf

8. Albornos-Muñoz L, González-María E, Moreno-Casbas T. Implantación de guías de buenas prácticas en España. Programa de centros comprometidos con la excelencia en cuidados. MedUNAB 2015; 17 (3): 163-169

9. Milán-Dobson N. Fundamentos de las rutas de cuidados en el paciente pediátrico. Rev Cubana Enfermer 2012; 28(2):181-188.

10. Díaz-Flores L, Durán-Villalobos M, Gallego-Pardo P, Gómez-Daza B, Gómez-Obando E, González-Acuña Y, et al. Análisis de los conceptos del modelo de adaptación de Callista Roy. Rev Aquichan. 2002; 2(1): 1923.Disponible en: http://aquichan.unisabana.edu.co/ index.php/aquichan/article/view/18/29

11. NANDA Internacional. Diagnósticos enfermeros. Definiciones y clasificación. 2012-2014. Madrid: Elsevier; 2012.

12. Moorhead S, Johnson M, Mass ML, Swanson E. Clasificación de resultados de enfermería (NOC). $4^{a}$ Edición. España: Elsevier. 2009.

13. Bulechek G, Butcher H, Dochterman J, Wagner C. Clasificación de intervenciones de enfermería (NIC). $6^{a}$ Edición. España: Elsevier. 2014.

14. Ley $911 / 2004$ de 5 de octubre. Responsabilidad deontológica para el ejercicio de la profesión de enfermería en Colombia; se establece el régimen disciplinario correspondiente y se dictan otras disposiciones. (Congreso de la república de Colombia). Disponible en: http://www.anec.org.co/images/ Documentos_ANEC/Ley911de2004.pdf

15. Resolución 8430/1993 de 4 de octubre. Normas científicas, técnicas y administrativas para la investigación en salud. (República de Colombia, Ministerio de Salud y Protección Social). Disponible en:https://www.invima.gov.co/images/pdf/medicamento s/resoluciones/etica_res_8430_1993.pdf

16. Ministerio de prōtección social y Organización Panamericana de Salud. Atención integrada a las enfermedades prevalentes de la infancia (AIEPI). Cuadro de procedimientos. 2010. Disponible en: http://www1.paho.org/col/dmdocuments/aiepi_cuadro_ procedimientos.pdf

17. Chueca MJ, Berrade S, Oyarzábal M. Talla baja y enfermedades raras. Anales Sis San Navarra 2008; 31(2): 31-53.

18. Rigotti-Baltor MR, Dupas G. Experiences from families of children with cerebral paralysis in context of social vulnerability. Rev. Latino-Am. Enfermagem, 2013; 21(4): 956-963.

19. Grau C, Fernández M. Familia y enfermedad crónica pediátrica. Anales Sis San Navarra. 2010; 33(2): 203212.

20. Romero-Guevara SL, Villamizar-Carvajal B, RuedaDíaz LJ. Apoyo telefónico: Una estrategia de intervención para cuidadores familiares de personas con enfermedad crónica. Rev. Univ. Ind. Santander. 2011; 43(2): 191-201. 